\title{
Are infusion phlebitis assessment scales reliable and valid? Gillian Ray-Barruel
}

\author{
Ray-Barruel G, Polit DF, Murfield JE, Rickard CM. "Infusion Phlebitis Assessment \\ Measures: A Systematic Review.” Journal of Evaluation in Clinical Practice (2014); 20(2): \\ 191-202. doi: 10.1111/jep.12107
}

Background: Having heard of several different infusion phlebitis assessment scales we wondered which scale was the most reliable and valid to recommend to our colleagues in clinical practice. That started a journey that would take 18 months and open more than one can of worms.

Methods: We decided to conduct a literature search to locate any study that reported phlebitis as an outcome measure. Originally we had no idea what we might find. Perhaps 5 or 10 different ways of measuring phlebitis? We searched MEDLINE, CINAHL and the Cochrane Database of Systematic Reviews until September 2013, and we included any research article that reported phlebitis by using either a scoring scale or definition.

Results: A total of 233 papers reported measuring phlebitis as an outcome measure: 101 used a scale, 79 used a definition only and 53 of the papers did not give any definition of phlebitis. Incredibly we found 71 different phlebitis scales using different combinations, definitions and cut-off points for 15 discrete symptoms. Some studies considered phlebitis when the patient had one symptom, others used 2 or 3, or even 4 symptoms. Only erythema was reported as a phlebitis symptom in every study involving a scale or definition, but even this varied in the ways it could be measured.

Many scales were available in several versions and authors did not always report which version they used. Many authors modified an existing scale without then testing the psychometric properties of the tool they were using.

Consequently phlebitis prevalence rates reported ranged from $0-91 \%$ for studies using a scale, and $0-100 \%$ for studies using a definition alone. Some studies reported phlebitis rates per patient, others reported rates per cannula, other reported rates per catheter days.

A handful of papers reported assessing their phlebitis tool for reliability or validity, so our nursing colleague and renowned statistician Prof Denise Polit performed an analysis of these findings using the COSMIN (COnsensus-based Standards for the selection of health Measurement Instruments) guidelines. Despite several authors reporting on reliability or validity, the assessments conducted and statistical scores were often inadequate when compared to the recommendations for psychometric testing.

Conclusion: At this time, no published phlebitis scale has been thoroughly validated for use in the clinical setting. The enormous disparities in definitions and ways of measuring and reporting phlebitis contribute to the extreme variations in phlebitis prevalence rates. The Infusion Nurses Society (INS) recommends phlebitis rates should be less than 5\%, but until phlebitis can be measured and reported in a consistent way this seems an arbitrary goal. As infusion phlebitis can have serious consequences for the patient, including catheter-associated bloodstream infection and prolonged hospitalisation, further research into assessment of phlebitis is urgently needed.

Key points

- There are dozens of phlebitis assessment scales in use, but none has been thoroughly validated for use in the clinical setting. 
- The broad disparities in measuring and reporting phlebitis have lead to enormous variations in reported phlebitis prevalence rates.

- Further research into a reliable and valid way of measuring phlebitis is urgently needed. 